\title{
Hydrogen peroxide induces nucleus pulposus cell apoptosis by ATF4/CHOP signaling pathway
}

\author{
YI LIU \\ Department of Orthopedics, The First People's Hospital of Fuyang District, Hangzhou, Zhejiang 311400, P.R. China
}

Received February 11, 2020; Accepted June 19, 2020

DOI: $10.3892 /$ etm.2020.9052

\begin{abstract}
Oxidative stress induces excessive apoptosis resulting in the reduction of intervertebral disc cells, the consequent reduction of extracellular matrix (ECM) synthesis, and compositional changes, which is the pathological basis for intervertebral disc degeneration (IVDD). The present study explored the activating transcription factor 4 (ATF4)/C/EBP homologous protein (CHOP) signaling pathway in the $\mathrm{H}_{2} \mathrm{O}_{2}$-induced nucleus pulposus (NP) cell apoptosis. Human degenerated intervertebral discs were collected from Lumbar disc surgery. NP cells isolated from the tissues were cultured with $\mathrm{H}_{2} \mathrm{O}_{2}$ to induce apoptosis in vitro. Malondialdehyde (MDA) analysis was performed to determine the reactive oxygen species (ROS) of the tissue. Western blot analysis and reverse transcription-polymerase chain reaction (RT-PCR) were performed to analyze collagen II, ATF4, CHOP, and caspase-9 gene expression. Flow cytometry was used to determine the apoptotic ratio of NP cells. siRNA was also used to silence ATF4 and CHOP gene expression. NP tissues in higher degenerated degree underwent much more MDA, expressed less collagen II, more ATF4, CHOP, and caspase- 9 compared with the mildly degenerated tissues. $\mathrm{H}_{2} \mathrm{O}_{2}$ induced NP cell apoptosis by upregulating expression of ATF4, CHOP and caspase-9. The silencing of ATF4 or CHOP alleviated NP cell apoptosis by suppressing caspase-9 expression. Inhibiting caspase-9 did not affect ATF4 and CHOP expression but protected NP cells from apoptosis. In this study, we found $\mathrm{H}_{2} \mathrm{O}_{2}$ could promote NP cell apoptosis by activating the ATF4/CHOP signaling pathway resulting in the upregulation of caspase-9. Interdict of ATF4, CHOP, or caspase-9 contributed to the reduction of apoptosis caused by $\mathrm{H}_{2} \mathrm{O}_{2}$.
\end{abstract}

Correspondence to: Dr Yi Liu, Department of Orthopedics, The First People's Hospital of Fuyang District, 429 Beihuan Road, Fuchun Street, Fuyang, Hangzhou, Zhejiang 311400, P.R. China E-mail: ma88u3@163.com

Key words: nucleus pulposus cells, apoptosis, ATF4, CHOP, oxidative stress

\section{Introduction}

Degenerative disc disease (DDD) refers to the biological degeneration of the intervertebral disc under various factors, which causes the changes in mechanical properties of the intervertebral disc, causing corresponding damage in adjacent bone joints and ligaments, affecting the spinal function and even compressing the spinal cord, nerve roots, and vertebral artery (1). It has gradually become the main cause of neck and low back pain, which not only reduces the quality of people's lives but also causes large Social and economic burdens. The current treatments of DDD are mainly conservative or surgical and not focusing on the cause of intervertebral disc degeneration (IVDD) but only relieve symptoms and some surgery may even lose adjacent segments (2). Generally, the intervertebral disc begins to degenerate around the age of 20 , and IVDD becomes increasingly more serious as age increases. Although the mechanism of IVDD has not been elucidated, current studies suggest that oxidative stress-induced nucleus pulposus (NP) cell apoptosis plays a crucial role in the development of IVDD (3).

In recent years, many studies have found that degeneration and aging of intervertebral discs are strictly related to the reduction of NP cells caused by apoptosis (4). Although intervertebral disc cells account for only $1 \%$ of the volume fraction of the intervertebral disc, these cells can produce factors that affect the synthesis of extracellular matrix (ECM), such as type I and II collagen, proteoglycans (PGs), prostaglandins, and nitric oxide, which play an essential role in maintaining the health of the intervertebral disc (5). Reactive oxygen species (ROS) is one of the most critical sources of oxidative stress, including harmful intermediates such as $02-, \mathrm{OH}^{-}$and $\mathrm{H}_{2} \mathrm{O}^{-}$. Typically, the body has a series of antioxidant defense systems: antioxidant enzymes, such as superoxide dismutase (SOD), glutathione peroxidase (GSH-Px), catalase; non-enzymatic molecules, such as thioredoxin, and vitamins A, C, E. The production and elimination of ROS are in a state of dynamic equilibrium (6).

When the balance between the generation of free radicals and the antioxidant defense system is broken, ROS accumulates resulting in cytotoxicity and causes tissue damage. Excessive ROS acts as a second messenger to trigger apoptotic signals, thereby modulating apoptosis by regulating the expression of apoptosis-related genes and the activation of nuclear transcription factors (7). High levels of ROS in the intervertebral disc are also indicated to induce apoptosis of NP cells and 
accelerate cell senescence to accelerate IVDD. Activating transcription factor 4 (ATF4) is a key transcription factor of the endoplasmic reticulum stress signaling pathway (8). It is stated that ATF4 is involved in the metabolism of amino acid and glucose, as well as intracellular antioxidant stress. ATF4 is also involved in the transcriptional regulation of a range of inflammatory factors (9). Overexpression of ATF4 can trigger a cascade of apoptotic factors that trigger apoptosis. Continuous overexpression of ATF4 promotes the upregulation of apoptosis-inducible genes, such as the transcription factor $\mathrm{C} / \mathrm{EBP}$ homologous protein $(\mathrm{CHOP})$, which ultimately leads to cell death (10). However, the role of ATF4 in IVDD and NP cell apoptosis caused by excess ROS is not clear. In this study, degenerative intervertebral disc specimens were collected, NP cells were isolated to explore the expression of apoptosis-related proteins including ATF4 and downstream $\mathrm{CHOP}$, in order to provide a theoretical basis for elucidating the role of ATF4 in oxidative stress-induced IVDD.

\section{Patients and methods}

Collection of patient tissue samples. In total 12 patients (aged 41 to 72 years, average age:53 years) who underwent lumbar disc herniation surgery in The First People's Hospital of Fuyang District (Hangzhou, China) from September to November 2018 were included in the study. The degree of IVDD in each operation section was determined according to the Pfirrmann classification score (11) based on the Magnetic Resonance Imaging (MRI), the degenerated degree was divided to grades I to $\mathrm{V}$. The tissues samples from the patients were grouped into i) Mild degenerated group (G2 and G3), and ii) Severe degenerated group (G4 and G5). The tissues were conserved in a sterile cell culture medium immediately after each operation for the following experiments. The study was approved by the Ethics Committee of The First People's Hospital of Fuyang District and signed informed consents were obtained from the patients and/or guardians. The study was conducted in accordance with the Declaration of Helsinki.

Isolation and cell culture of NP cells. The tissues in Pfirrmann classification grades 2 were washed with cold phosphate-buffered saline solution (PBS). Then the samples were cut into $0.3 \times 0.3 \mathrm{~cm}^{2}$ pieces and incubated with $0.25 \%$ type I collagenase at $37.5^{\circ} \mathrm{C}$ overnight for digesting. Cell pellets were filtrated with a strainer and re-suspended in Dulbecco's modified Eagle's medium (DMEM) (containing 10\% fetal bovine serum (FBS) and 1\% penicillin-streptomycin, Gibco; Thermo Fisher Scientific, Inc.). For cell treatment 50 and $100 \mu \mathrm{M} \mathrm{H}_{2} \mathrm{O}_{2}$ was used to induce ROS; siRNA transfection was used to silence ATF4 and CHOP gene expression; $20 \mu \mathrm{M}$ Z-LEHD-FMK (ZLF, a cell-permeable, irreversible caspase-9 inhibitor; purchased from Selleck) was used to inhibit the expression of caspase-9.

Malondialdehyde (MDA) analysis. The NP tissue was first lysed and homogenized using RIPA lysis buffer (Beyotime Institute of Biotechnology). Formulated TBA stock solution and MDA working fluid were prepared according to the instructions. Lysate $(0.1 \mathrm{ml})$ was used as a blank control, and a $0.1 \mathrm{ml}$ sample was added into a $0.2 \mathrm{ml}$ MDA test solution for measurement. Finally, the absorbance was measured at $532 \mathrm{~nm}$ using a microplate reader.

Cell transfection. NP cells were seeded in 6-well plates at a density of $2 \times 10^{5}$ to $4 \times 10^{5} /$ well before transfection. Transfection was performed using Lipofectamine 2000 (Invitrogen; Thermo Fisher Scientific, Inc.) + Opti MEM I lipofection according to the instructions. Negative control siRNA (null-siRNA), ATF4 siRNA, and CHOP siRNA were purchased from Invitrogen; Thermo Fisher Scientific, Inc. The medium was changed $48 \mathrm{~h}$ after transfection and cultured in a $37^{\circ} \mathrm{C}, 5 \% \mathrm{CO}_{2}$ incubator.

Western blot (WB) analysis. Protein extraction was performed on ice, and the reaction solution was pre-cooled with ice. The tissues or cells were washed with cold phosphate-buffered saline (PBS), lysed with radioimmunoprecipitation assay (RIPA) (Beyotime Institute of Biotechnology) lysis buffer, centrifuged (at 10,000 x g for $15 \mathrm{~min}$ at $4^{\circ} \mathrm{C}$ ), discarded, and resuspended in a cell lysate containing phosphatase inhibitor or protease inhibitor (Beyotime, Shanghai, China). Bicinchoninic acid (BCA) protein concentration assay kit (Beyotime Institute of Biotechnology) was used for the determination of protein quality. Then, the protein was added to the sodium dodecyl sulphate-polyacrylamide gel electrophoresis (SDS-PAGE) and transferred onto the polyvinylidene fluoride (PVDF) membranes (EMD Millipore). After 5\% milk blocking, membranes were coated with primary antibodies against: Collagen II (ab34712), ATF4 (ab31390), CHOP (ab240220), cleaved caspase-9 (ab2324), and $\beta$-actin (as loading control, ab179467) overnight at $4^{\circ} \mathrm{C}$. All the antibodies were purchased from Abcam. Membranes were then incubated with secondary antibody for $1 \mathrm{~h}$ at room temperature. Chemiluminescent ECL substrate (Beyotime Institute of Biotechnology) was used to expose the band.

Immunofluorescence (IF). NP cells were fixed with $4 \%$ paraformaldehyde (PFA) for $15 \mathrm{~min}$ and then incubated with $0.1 \%$ Triton $\mathrm{X}-100$ for $15 \mathrm{~min}$ at room temperature and blocked with $5 \%$ bovine serum albumin (BSA) for $1 \mathrm{~h}$ at room temperature. The cells were washed and incubated with primary antibodies against collagen II (ab34712; Abcam), caspase-9 (ab219590; Abcam) overnight at $4^{\circ} \mathrm{C}$. NP cells subsequently were incubated with Alexa Fluor 488 conjugated secondary antibody (Invitrogen; Thermo Fisher Scientific, Inc.) and 4',6-diamidino-2-phenylindole (DAPI) for $1 \mathrm{~h}$ in the dark. The staining intensity was measured by Image-Pro Plus software (Version X; Media Cybernetics).

Reverse transcription-polymerase chain reaction (RT-PCR). RT-PCR was performed to analyze the expression of related gene mRNA in NP cells. Total cellular RNA was extracted with RNAiso kit (Takara) according to the instructions, and reverse PrimeScript RT reagent kit with gDNA Eraser (Takara) was used for reverse transcription of RNA into cDNA. SYBR-Green Realtime PCR Master Mix (Toyobo) reagent was used for the RT-PCR reaction, and the system was carried out according to the instructions. Gene expression was calculated by normalization to glyceraldehyde 3-phosphate dehydrogenase (GAPDH) according to the method of $2^{-\Delta \Delta \mathrm{Ct}}$. The primers used for collagen II, ATF4, CHOP, and caspase- 9 are listed in Table I. 
Table I. Primer sequences of the genes for RT-PCR.

\begin{tabular}{lll}
\hline Gene name & \multicolumn{1}{c}{ Forward (5'-3') } & \multicolumn{1}{c}{ Reverse (5'-3') } \\
\hline Collagen II & TGGACGATCAGGCGAAACC & GCTGCGGATGCTCTCAATCT \\
ATF4 & ATGACCGAAATGAGCTTCCTG & GCTGGAGAACCCATGAGGT \\
CHOP & GGAAACAGAGTGGTCATTCCC & CTGCTTGAGCCGTTCATTCTC \\
Caspase-9 & CTTCGTTTCTGCGAACTAACAGG & GCACCACTGGGGTAAGGTTT \\
GAPDH & ACAACTTTGGTATCGTGGAAGG & GCCATCACGCCACAGTTTC
\end{tabular}

RT-PCR, quantitative reverse-transcription polymerase chain reaction; ATF4, activating transcription factor 4; CHOP, C/EBP homologous protein; GAPDH, glyceraldehyde 3-phosphate dehydrogenase.

A

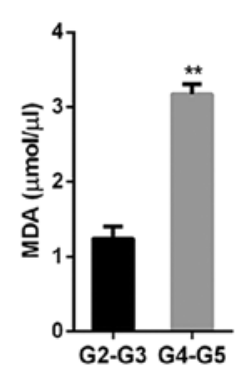

C

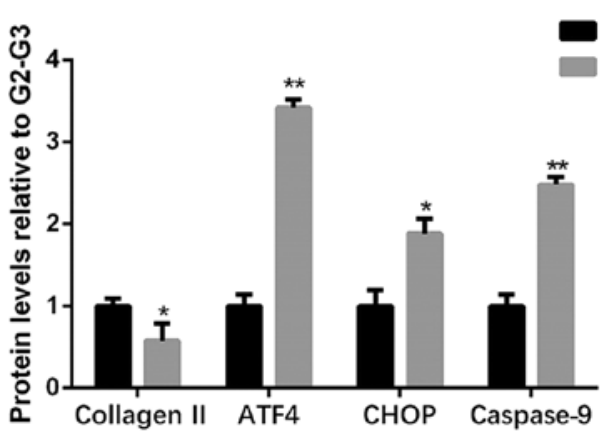

B

G2-G3

G4-G5

Figure 1. ROS and apoptosis levels in human degenerated intervertebral disc tissues. Tissues were lysed and ROS measured with MDA methods. Total protein and mRNA were extracted from the tissues in mild (G2-G3) and severe (G4-G5) degeneration. (A) MDA level of different degenerated degrees of disc tissues. (B and C) The protein levels of collagen II, ATF4, CHOP and caspase-9 were determined by (B) Western blot and (C) quantification analysis. (D) mRNA levels of collagen II, ATF4, CHOP and caspase- 9 were determined by RT-PCR. The values are mean $\pm \mathrm{SD}$ of three independent experiments ( $=3$ ). ( $\mathrm{P}<0.05$, ${ }^{* *} \mathrm{P}<0.01,{ }^{* * *} \mathrm{P}<0.001$, compared with G2-G3 group). ROS, reactive oxygen species; ATF4, activating transcription factor 4; CHOP, C/EBP homologous protein; RT-PCR, reverse transcription-polymerase chain reaction.

Flow cytometry. NP cells were seeded in a 6-well plate. After undergoing different treatments, the cells were collected at a density of $\sim 1 \times 10^{6} /$ well. The cells were washed with cold PBS and mixed with $5 \mu \mathrm{l} \mathrm{PI}$ and $5 \mu \mathrm{l}$ Annexin $\mathrm{V}$ according to the Annexin V-PI double staining test kit (Keygen). Following incubating at room temperature for $15 \mathrm{~min}$ in the dark, the apoptosis rate of each group was measured by flow cytometry. The total apoptosis ratio was determined by the collection of both early and late apoptotic cells.

Statistical analysis. All statistical analyses were performed using Statistical Product and Service Solutions (SPSS) 13.0 statistical software (SPSS Inc.). Data were expressed as the mean \pm SD (standard deviation). Differences between two groups were analyzed by using the paired Student's t-test. A comparison between multiple groups was made using a One-way analysis of variance (ANOVA) test followed by the Post Hoc Test (Least Significant Difference). $\mathrm{P}<0.05$ was considered to indicate a statistically significant difference.

\section{Results}

Human intervertebral disc degenerates combining with increased ROS and apoptosis. We collected degenerated intervertebral disc tissues from the patients undergoing herniated disc surgery and divided them into two groups according to the Pfirrmann score of disc degeneration. Most patients with IDD who need surgery in the clinic are highly degenerative. It is difficult for us to obtain a disc without any degeneration. Therefore, we used the highly degenerated specimens to compare with the mildly degenerated specimens. To determine the level of oxygen radicals in the mild and severe degenerated disc samples, MDA assay was performed, and the data showed a higher degree of degeneration expressed the MDA level (Fig. 1A). Collagen II is 

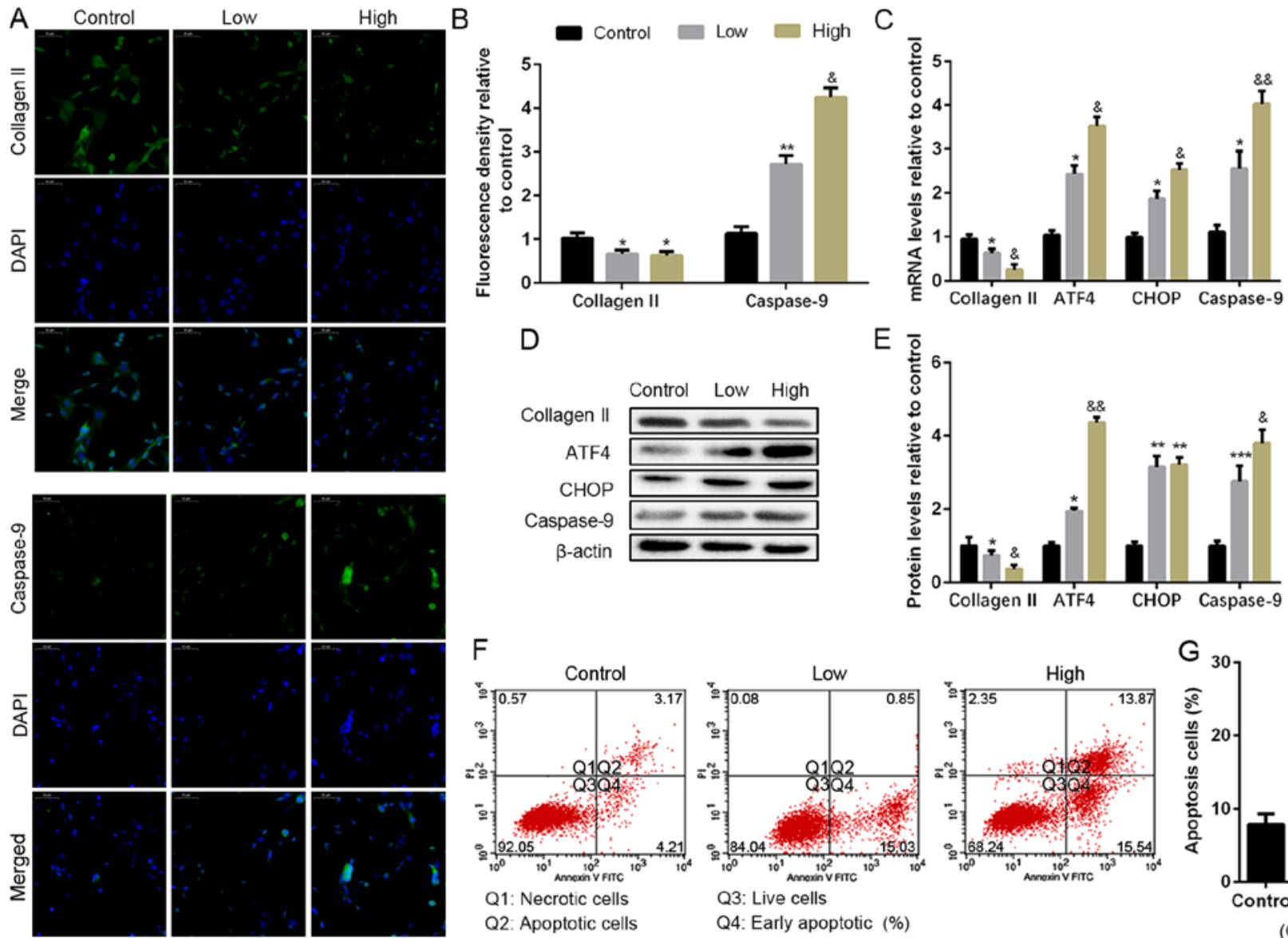

$\mathrm{E}$

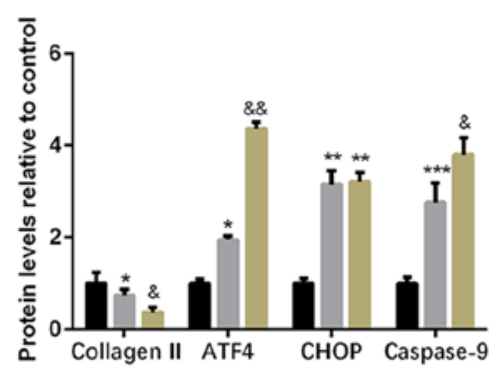

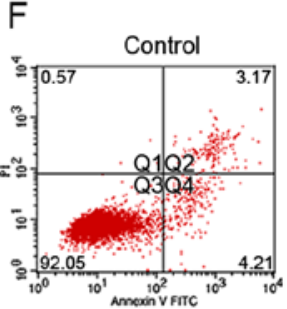

Q1: Necrotic cells Q2: Apoptotic cells
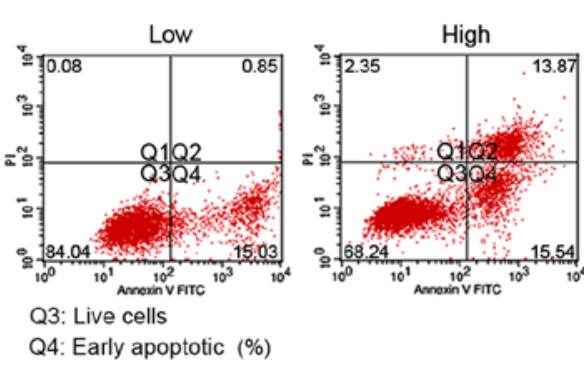

Figure 2. Apoptosis levels in $\mathrm{H}_{2} \mathrm{O}_{2}$ induce NP cells in vitro. NP cells of $\mathrm{G} 2$ tissues were cultured with a low or high concentration of $\mathrm{H}_{2} \mathrm{O}_{2}(50$ or $100 \mu \mathrm{M})$ for $24 \mathrm{~h}$. (A and B) The protein expression level of collagen II and caspase-9 were determined by (A) IF (magnification, $\mathrm{x} 400$ ) and (B) quantification analysis. (C) The mRNA expression levels of collagen II, ATF4, CHOP, and caspase-9 were assayed by RT-PCR. (D and E) The protein expression levels of collagen II, ATF4, CHOP and caspase-9 were determined by (D) WB and (E) quantification analysis. (F and G) The ratio of apoptotic cells was analyzed by (F) flow cytometry and $(\mathrm{G})$ quantification analysis. The values are mean $\pm \mathrm{SD}$ of three independent experiments $(\mathrm{n}=3)$. $\left({ }^{*} \mathrm{P}<0.05,{ }^{* *} \mathrm{P}<0.01,{ }^{* * *} \mathrm{P}<0.001, \mathrm{compared}\right.$ with control; ${ }^{\&} \mathrm{P}<0.05$, \& ${ }^{\&} \mathrm{P}<0.01$, compared with $50 \mu \mathrm{M} \mathrm{H}_{2} \mathrm{O}_{2}$ ). ATF4, activating transcription factor 4; CHOP, C/EBP homologous protein; WB, western blot; NP, nucleus pulposus; RT-PCR, reverse transcription-polymerase chain reaction; IF, immunofluorescence.

the main protected element of ECM secreted by the NP cells that decreased with the degeneration of NP cells (12). The result of western blot analysis indicated the severely degenerated discs expressed a lower content of collagen II and an increased level of ATF4, CHOP, and caspase-9 compared with the mild group (Fig. 1B and C). RT-PCR was also used to analyze the mRNA expression of collagen II, ATF4, CHOP, and caspase-9 of the intervertebral disc samples, and the results were totally parallel to the protein levels (Fig. 1D). The data suggest that severely degenerative disc is in a higher state of oxidative stress and a severe level of apoptosis related to the activation of ATF4/CHOP/caspase-9.

$\mathrm{H}_{2} \mathrm{O}_{2}$ treatment increases $\mathrm{NP}$ cell apoptosis by ATF4/CHOP and caspase-9 upregulation. To explore the effect of excess ROS in the progress of NP cell degeneration, different concentrations of $\mathrm{H}_{2} \mathrm{O}_{2}(50$ or $100 \mu \mathrm{M})$ were used as a source of oxygen radicals. As shown in Fig. 2A and B, the expression of collagen II was decreased, resulting from the $\mathrm{H}_{2} \mathrm{O}_{2}$ treatment; in addition, higher concentration caused more reduction of collagen II. Besides, $\mathrm{H}_{2} \mathrm{O}_{2}$ promoted caspase-9, the apoptosis maker, of NP cells as well. Moreover, the mRNA and protein levels of collagen II, ATF4, CHOP and caspase-9 were also measured by RT-PCR and WB (Fig. 2C-E). The data indicated that collagen II was reduced caused by $\mathrm{H}_{2} \mathrm{O}_{2}$ treatment with a dose-dependence compared with the control; ATF4 and CHOP expression were increased, which were more significant in the high dose $\mathrm{H}_{2} \mathrm{O}_{2}$. In addition, $\mathrm{H}_{2} \mathrm{O}_{2}$ also activated caspase- 9 expression compared with the control. Flow cytometry was also used to measure apoptotic cells, and the result indicated a higher apoptotic population after $\mathrm{H}_{2} \mathrm{O}_{2}$ treatment (Fig. 2F and $\mathrm{G}$ ). The results suggested $\mathrm{H}_{2} \mathrm{O}_{2}$ treatment promoted NP cell degeneration and apoptosis, which might be related to the upregulation of ATF4/CHOP and caspase-9.

Silencing of ATF4 alleviates $\mathrm{H}_{2} \mathrm{O}_{2}$-induced $\mathrm{NP}$ cells apoptosis. To determine the effect of ATF4 in $\mathrm{H}_{2} \mathrm{O}_{2}$-induced NP cell apoptosis, the ATF4 gene was silenced via transfecting with siRNA targeting ATF4, and null-siRNA was used as a negative control. The non-transfected NP cells and the siRNA transfected NP cells were cultured with $100 \mu \mathrm{M} \mathrm{H}_{2} \mathrm{O}_{2}$ for $24 \mathrm{~h}$. Silencing of ATF4 played a protective effect on collagen II expression in the treatment of $\mathrm{H}_{2} \mathrm{O}_{2}$. Furthermore, IF also showed ATP4 deficiency reduced the caspase- 9 expression compared with the $\mathrm{H}_{2} \mathrm{O}_{2}$ group, which was also confirmed in the WB and RT-PCR (Fig. 3A and B). The silencing of 

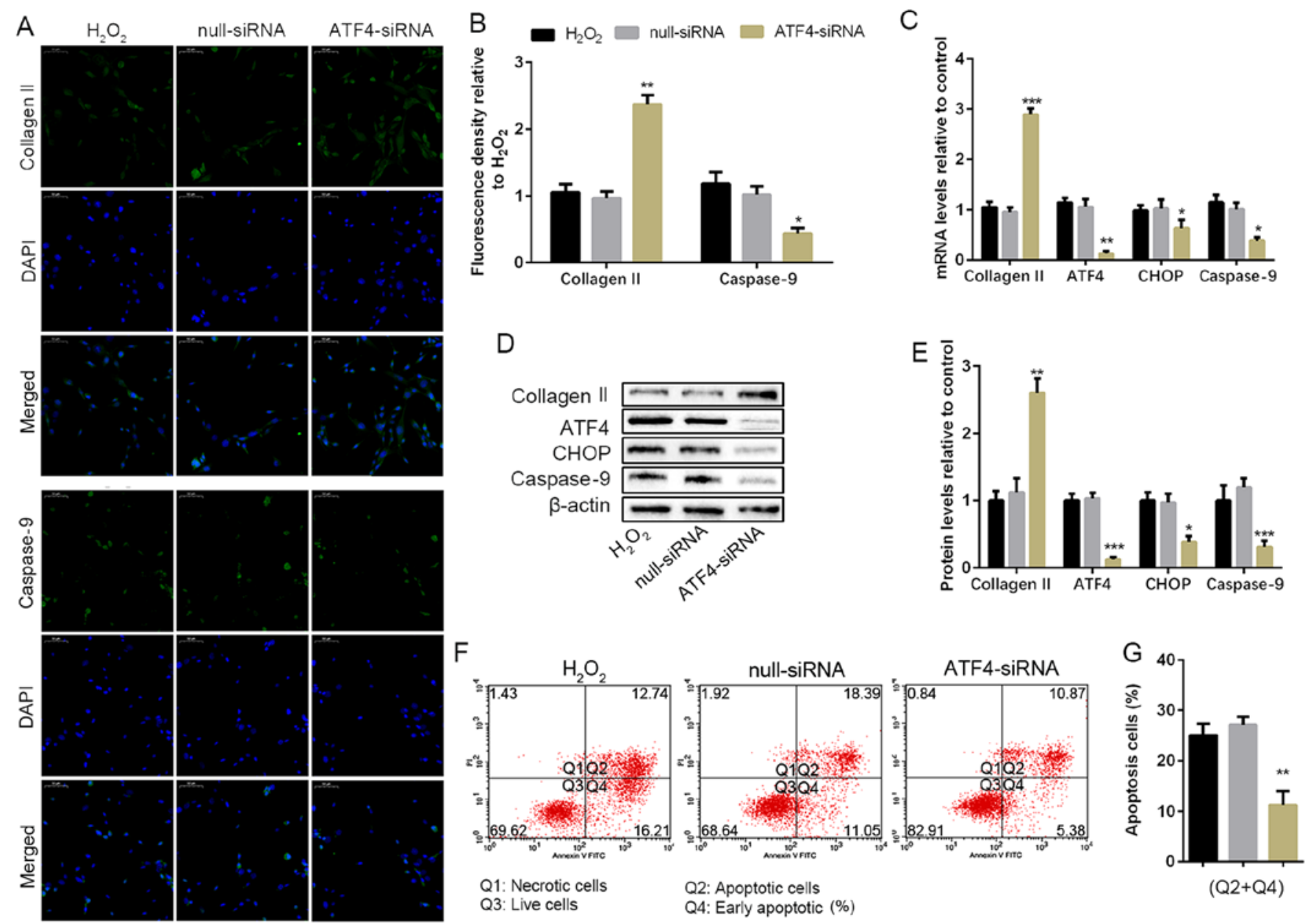

Figure 3. ATF4 deficiency weakens apoptosis and protects human NP cell degeneration. NP cells of G2 tissues without or with the ATF4-siRNA transfection were cultured with $100 \mu \mathrm{M}$ of $\mathrm{H}_{2} \mathrm{O}_{2}$ for $24 \mathrm{~h}$. (A and B) The protein expression level of collagen II and caspase- 9 were determined by (A) IF (magnification, $\mathrm{x} 400$ ) and (B) quantification analysis. (C) The mRNA expression levels of collagen II, ATF4, CHOP and caspase-9 were assayed by RT-PCR. (D and E) The protein expression levels of collagen II, ATF4, CHOP and caspase-9 were determined by (D) WB and (E) quantification analysis. (F and G) The ratio of apoptotic cells was analyzed by $(\mathrm{F})$ flow cytometry and $(\mathrm{G})$ quantification analysis. The values are mean $\pm \mathrm{SD}$ of three independent experiments $(\mathrm{n}=3)$. $\left({ }^{*} \mathrm{P}<0.05\right.$, ${ }^{* *} \mathrm{P}<0.01$, ${ }_{* * * *} \mathrm{P}<0.001$, compared with $100 \mu \mathrm{M} \mathrm{H}_{2} \mathrm{O}_{2}$ ). ATF4, activating transcription factor 4; CHOP, C/EBP homologous protein; WB, western blot; NP, nucleus pulposus; RT-PCR, reverse transcription-polymerase chain reaction; IF, immunofluorescence.

ATF4 contributed to the decrease of CHOP mRNA expression, which could also be responsible for the reduction of caspase-9 (Fig. 3C). WB was also performed to analyze the protein expression of $\mathrm{CHOP}$, which was consistent with the result of RT-PCR (Fig. 3D and E). The data of flow cytometry also confirmed silencing of ATF4 decreased the apoptotic cells compared with the $\mathrm{H}_{2} \mathrm{O}_{2}$ group (Fig. 3F and G). In brief, the silencing of ATF4 blocked the ATF4/CHOP pathway for the activation of downstream gene caspase-9, which resulted in the reduction of NP cell apoptosis.

Silencing of CHOP alleviates $\mathrm{H}_{2} \mathrm{O}_{2}$-induced $\mathrm{NP}$ cell apoptosis. To determine the effect of CHOP, the downstream target of ATF4, in $\mathrm{H}_{2} \mathrm{O}_{2}$-induced $\mathrm{NP}$ cell apoptosis, the CHOP gene was blocked by transfecting with CHOP-siRNA. The non-transfected NP cells and the siRNA transfected $\mathrm{NP}$ cells were cultured with $100 \mu \mathrm{M} \mathrm{H}_{2} \mathrm{O}_{2}$ for $24 \mathrm{~h}$. However, the silencing of $\mathrm{CHOP}$ significantly upregulated collagen II protein expression and decreased the caspase-9 expression compared with the $\mathrm{H}_{2} \mathrm{O}_{2}$ group (Fig. 4A and B). Similar results were obtained from the RT-PCR and WB analysis (Fig. 4C-E). Furthermore, both the mRNA and protein expression of $\mathrm{CHOP}$ were significantly reduced resulting from siRNA transfection, but $\mathrm{CHOP}$ silencing did not affect ATF4 expression (Fig. 4C-E). The number of apoptotic NP cells was obviously reduced compared with the $\mathrm{H}_{2} \mathrm{O}_{2}$ group when the $\mathrm{CHOP}$ gene was silenced through flow cytometry assay (Fig. 4F and G). The result indicated silencing of CHOP broke the ATF4/CHOP pathway for the activation of caspase- 9 , which contributed to the reduction of NP cell apoptosis in the $\mathrm{H}_{2} \mathrm{O}_{2}$ situation.

Inhibiting caspase-9 alleviates $\mathrm{H}_{2} \mathrm{O}_{2}$-induced $\mathrm{NP}$ cell apoptosis. Previous results of this study suggested caspase- 9 was activated by ATF4/CHOP pathway. It was investigated whether the change of caspase-9 levels would affect ATF4 or CHOP expression and the degree of NP cell apoptosis caused by $\mathrm{H}_{2} \mathrm{O}_{2}$. ZLF $(13,14)$, a widely used inhibitor of caspase- 9 was used to pretreat NP cells for $24 \mathrm{~h}$. The chemical structural formula of ZLF is shown in Fig. 5A. ZLF pretreated NP cells showed a protected role of collagen II production compared with the non-pretreated group (Fig. 5B-E). Under 
A
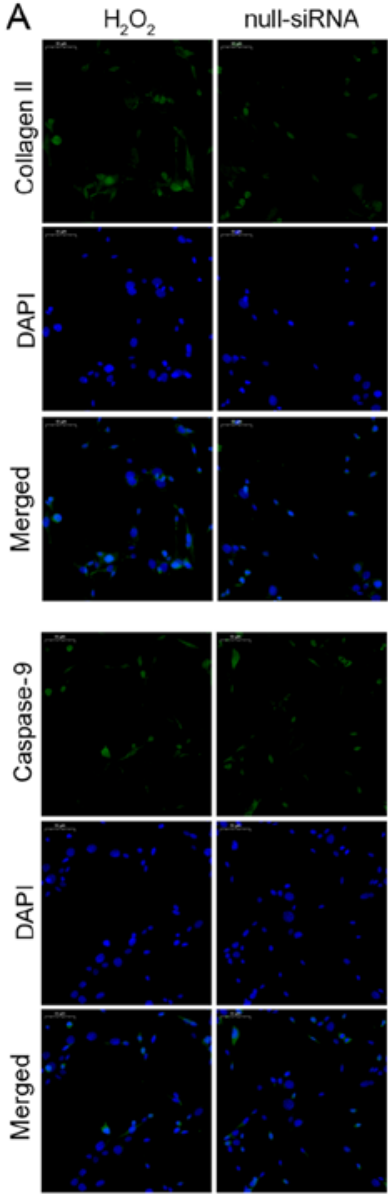

B

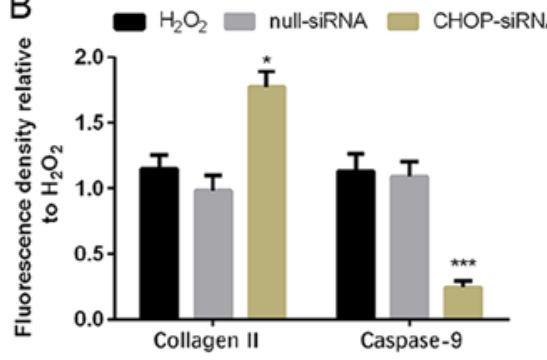

D

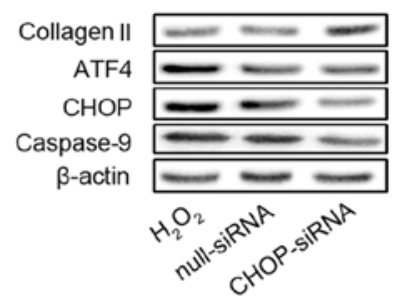

$\mathrm{F}$

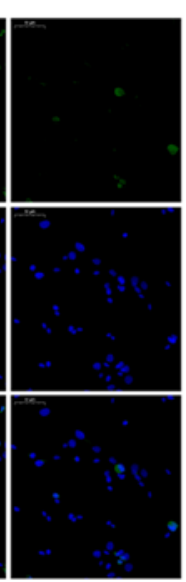

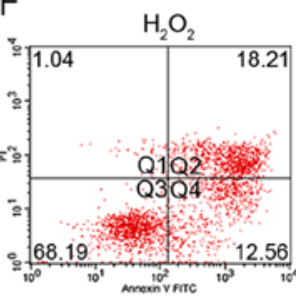

Q1: Necrotic cells Q2: Apoptotic cells

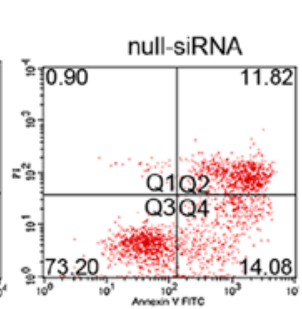

Q4: Early apoptotic (\%)
Q3: Live cells
C

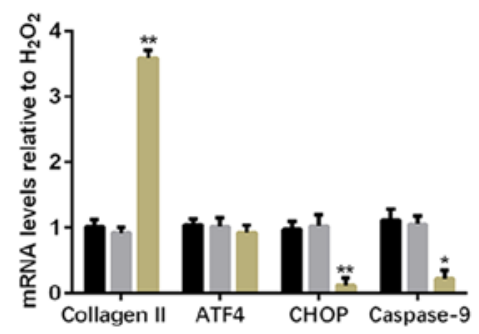

E ô
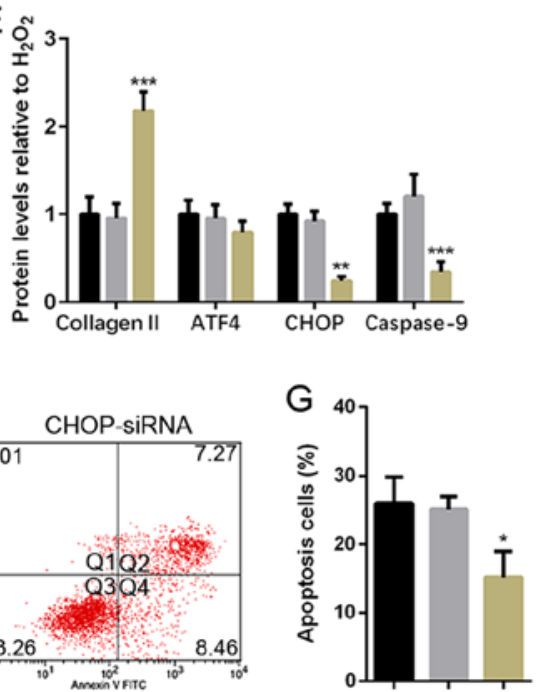

(Q2+Q4)

Figure 4. CHOP deficiency weakens apoptosis and protects human NP cell degeneration. NP cells of G2 tissues without or with the CHOP-siRNA transfection were cultured with $100 \mu \mathrm{M}$ of $\mathrm{H}_{2} \mathrm{O}_{2}$ for $24 \mathrm{~h}$. (A and B) The protein expression level of collagen II and caspase-9 were determined by (A) IF (magnification, $\mathrm{x} 400$ ) and (B) quantification analysis. (C) The mRNA expression levels of collagen II, ATF4, CHOP and caspase-9 were assayed by RT-PCR. (D and E) The protein expression levels of collagen II, ATF4, CHOP, and caspase-9 were determined by (D) WB and (E) quantification analysis. (F and G) The ratio of apoptotic cells was analyzed by $(\mathrm{F})$ flow cytometry and $(\mathrm{G})$ quantification analysis. The values are mean $\pm \mathrm{SD}$ of three independent experiments $(\mathrm{n}=3)$. $\left({ }^{*} \mathrm{P}<0.05,{ }^{* *} \mathrm{P}<0.01\right.$, ${ }^{*}{ }^{* * *} \mathrm{P}<0.001$, compared with $100 \mu \mathrm{M} \mathrm{H}_{2} \mathrm{O}_{2}$ ). ATF4, activating transcription factor 4; CHOP, C/EBP homologous protein; WB, western blot; NP, nucleus pulposus; RT-PCR, reverse transcription-polymerase chain reaction; IF, immunofluorescence.

the condition of caspase-9 suppression, we did not achieve a significant difference in the protein expression of ATF4 and CHOP, indicating that the level of caspase- 9 cannot affect ATF4/CHOP expression (Fig. 5D and E). Moreover, as a result of the suppression of caspase-9, the apopotic NP cell population was lower than the $\mathrm{H}_{2} \mathrm{O}_{2}$ group (Fig. $5 \mathrm{~F}$ and $\mathrm{G}$ ). In short, caspase-9 is a downstream target of the ATF4/CHOP pathway in the NP cells, and ZLF is effective in suppressing apoptosis in $\mathrm{H}_{2} \mathrm{O}_{2}$-treated NP cells.

\section{Discussion}

The expression of ROS has been confirmed to be increased during the progress of IVDD, suggesting that ROS may play an essential role in the pathological process of IVDD (15). In the present study, the intervertebral disc with severe degenerated degree expressed a higher level of MDA compared with the mild, suggesting the degree of degeneration is positively related to the content of ROS. Apoptosis is the natural physiological process of the body as well as cell production. Both coexist and maintain dynamic balance. This balance is a necessary condition for maintaining the morphological stability of the body tissues and organs, the physiological functions of the body, and the security of the internal and external environment. If the cell apoptosis is too much or too little, this balance damage will lead to disease. Excessive apoptosis of NP cells is the direct cause of IVDD (16). Ha et al (17) found that the cell apoptosis rate of patients with intervertebral disc prolapse was $74.3 \%$, disc herniation was $42.8 \%$, while the regular control group was only $28 \%$. Zhao et al (18) confirmed that various stimulating factors could induce apoptosis of intervertebral disc cells and further cause the reduction of intervertebral disc matrix and promote the IVDD process. Numbers of studies have elucidated that low levels of oxygen free radicals can induce apoptosis. At the same time, ROS, such as $\mathrm{O}_{2}^{-}, \mathrm{H}_{2} \mathrm{O}_{2}$, lipid peroxide, and $\mathrm{NO}$, is considered to be widely involved in apoptosis. As shown in our results, apoptosis-related ATF4, CHOP and caspase-9 were significantly decreased in the severely degenerated disc tissues. Therefore, ROS is suggested to be related to apoptosis in the degenerated intervertebral disc.

Collagen II, secreted by NP cells, is one of the most important components of the extracellular matrix, and its content is an important indicator of whether NP cells are 
A

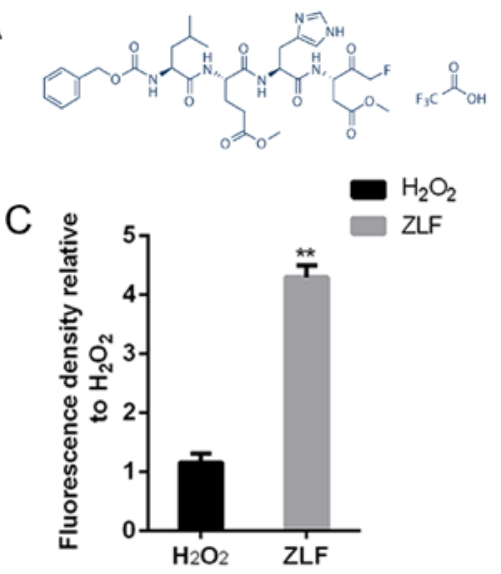

$\mathrm{D}$

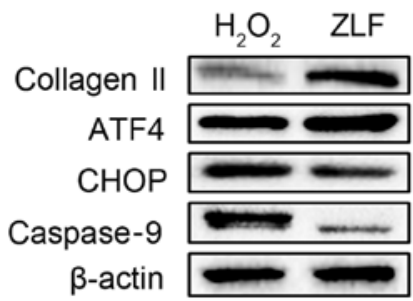

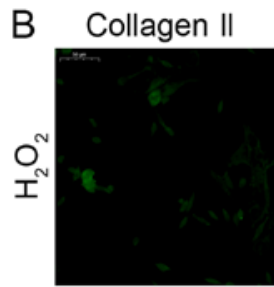
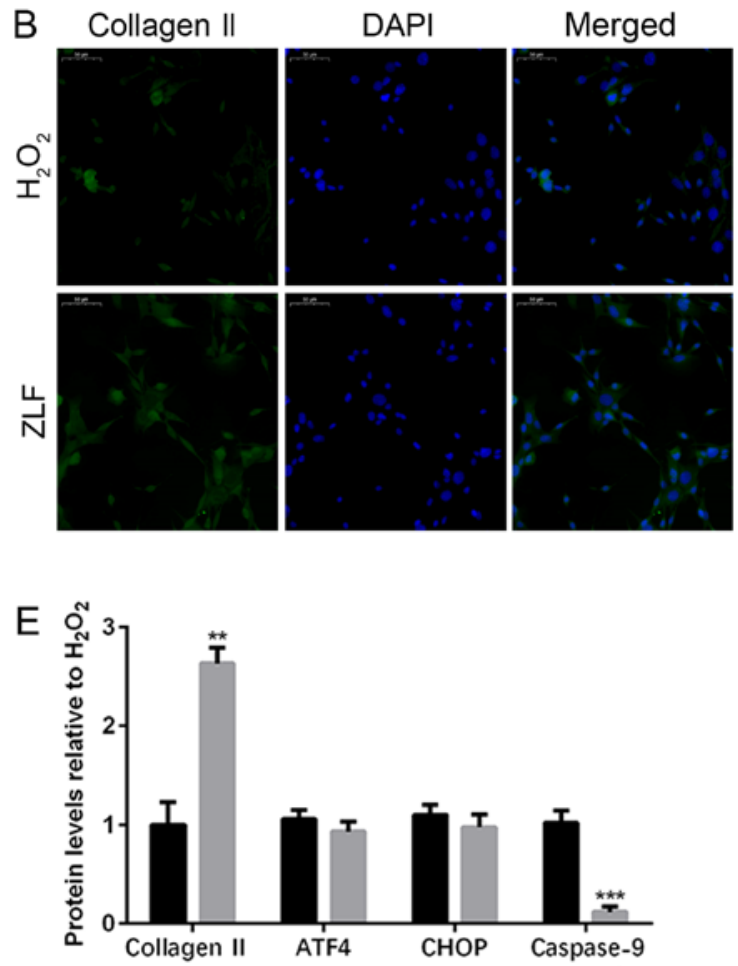

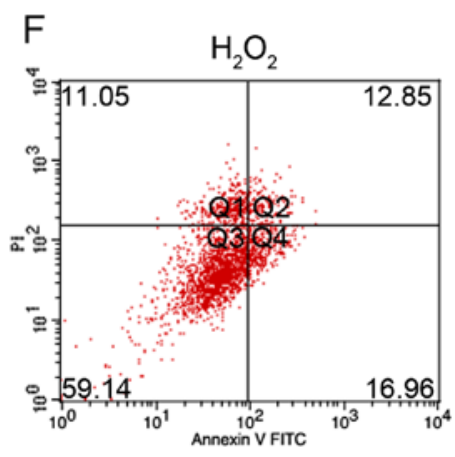

Q1: Necrotic cells

Q2: Apoptotic cells

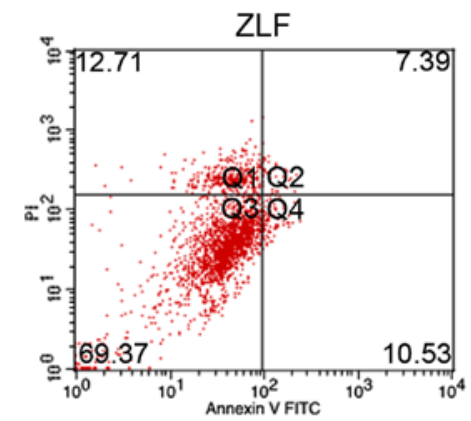

Q3: Live cells

Q4: Early apoptotic (\%)
G

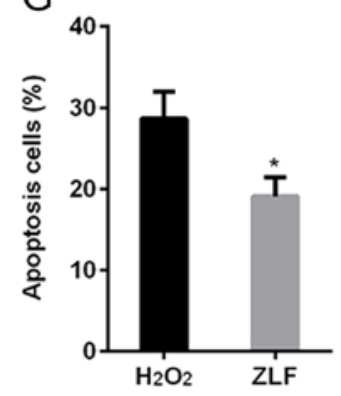

(Q2+Q4)

Figure 5. ZLF inhibits apoptosis and protects human NP cell degeneration. NP cells of G2 tissues were pretreated with ZLF $(20 \mu \mathrm{M})$ for $24 \mathrm{~h}$. Then, NP cells without or with ZLF pretreatment were cultured with $100 \mu \mathrm{M}$ of $\mathrm{H}_{2} \mathrm{O}_{2}$ for $24 \mathrm{~h}$. (A) The chemical structural formula of ALF. (B and C) The protein expression level of collagen II was determined by (B) IF (magnification, x400) and (C) quantification analysis. (D and E) The protein expression levels of collagen II, ATF4, CHOP and caspase-9 were determined by (D) WB and (E) quantification analysis. (F and G) The ratio of apoptotic cells was analyzed by (F) flow cytometry and $(\mathrm{G})$ quantification analysis. The values are mean $\pm \mathrm{SD}$ of three independent experiments $(\mathrm{n}=3) .\left({ }^{*} \mathrm{P}<0.05,{ }^{* *} \mathrm{P}<0.01,{ }^{* * * *} \mathrm{P}<0.001\right.$, compared with $100 \mu \mathrm{M} \mathrm{H}_{2} \mathrm{O}_{2}$ ). ATF4, activating transcription factor 4; CHOP, C/EBP homologous protein; WB, western blot; NP, nucleus pulposus; IF, immunofluorescence.

functional. In this experiment, $\mathrm{H}_{2} \mathrm{O}_{2}$ was used to upregulate ROS in inducing apoptosis of NP cells (19). As the concentration of $\mathrm{H}_{2} \mathrm{O}_{2}$ increased, the expression of collagen II in NP cells decreased, but apoptosis level increased significantly, along with the upregulation of ATF4, CHOP and caspase-9 expression. A large number of reports have reported that ROS participate in cell apoptosis by activating ATF4/CHOP pathway. Xian et al (20) stated ROS activates apoptosis in adriamycin-treated, bortezomib sensitized human osteosarcoma cells via p-eIF2 $\alpha / \mathrm{ATF} 4 / \mathrm{CHOP}$ axis. Zong et al (21) reported that upregulating ATF4 expression could increase cellular ROS and the sensitivity to apoptosis. ATF4 is upregulated by stress signals containing oxidative stress, hypoxia, and endoplasmic reticulum stress. ATF4 also affects the expression of genes involved in oxidative stress (22). The ATF4 gene expression was silenced resulting in the downregulation of both CHOP and caspase-9, reversing the negative effect of $\mathrm{H}_{2} \mathrm{O}_{2}$ on the NP cells. In addition, CHOP, a transcription factor, is expressed at deficient levels under normal conditions and is significantly elevated in endoplasmic reticulum stress through the activation of ATF4 (23). After suppressing the expression of CHOP by gene silencing, caspase-9 and apoptotic cell population were also obviously reduced without affecting ATF4. They contributed to upregulation of the expression of collagen II under conditions of oxidative stress, which confirms CHOP as the downstream gene of ATF4 in the apoptosis-related progress.

Among the CHOP-mediated endoplasmic reticulum stress-responsive apoptotic pathways, the upstream regulation mechanism is basically precise, but its downstream mechanism remains to be further studied. The present study indicates that the target genes of CHOP may include $\mathrm{Bcl}-2$, TRB3, GADD34 and DOCs $(24,25)$. Regardless of which targets downstream of CHOP, activated caspase- 9 will be 
initiated to trigger apoptosis at the end $(26,27)$. Regardless of silencing by ATF4 or CHOP in $\mathrm{H}_{2} \mathrm{O}_{2}$ treated NP cells, apoptosis was decreased significantly as well as the caspase- 9 level in the present study. As previously noted, ROS mediated caspase-9 activation increases apoptosis of EBV-transformed B cells (28); in addition, inhibiting caspase-9 is efficacious to suppress apoptosis $(29,30)$. ZLF is a novel specific inhibitor of caspase-9 (31). After ZLF treatment, NP cells expressed a raised level of collagen II and the apoptotic cell population decreased. Though ZLF did not affect the expression of ATF4 and CHOP, it achieved the same result in reversing the $\mathrm{H}_{2} \mathrm{O}_{2}$ induced apoptosis.

In conclusion, this study recovered, for the first time, crosstalk between CHOP and ATF4 in the development of IDD, especially under the $\mathrm{H}_{2} \mathrm{O}_{2}$ mediated high-ROS situation, which involves caspase- 9 related apoptosis. With the aggravation of IVDD, the ATF4/CHOP pathway is activated, resulting in apoptosis of NP cells. Blocking ATF4 or $\mathrm{CHOP}$ can partly suppress caspase-9 and alleviate ROS related NP cell apoptosis, which also protects NP cell degeneration. Suppressing the expression of caspase- 9 by ZLF can protect NP cells from apoptosis. In conclusion, $\mathrm{H}_{2} \mathrm{O}_{2}$ induces NP cell apoptosis by ATF4/CHOP signaling pathway, and blocking ATF4/CHOP or caspase-9 can protect NP cell degeneration via apoptosis suppression. Additionally, to improve the limitation and verify the findings of the present study, we plan to use siRNA to suppress the expression of caspase- 9 and establish animal model to set up a non-degenerative NP tissue control group in the next project. Additionally, human studies with a control group are also required to verify the findings of this study.

In conclusion, in this study, $\mathrm{H}_{2} \mathrm{O}_{2}$ was found to promote NP cell apoptosis by activating the ATF4/CHOP signaling pathway resulting in the upregulation of caspase-9. Interdict of ATF4, CHOP, or caspase-9 contributed to the reduction of apoptosis caused by $\mathrm{H}_{2} \mathrm{O}_{2}$.

\section{Acknowledgements}

Not applicable.

\section{Funding}

No funding was received.

\section{Availability of data and materials}

All data generated or analyzed during this study are included in this published article.

\section{Authors' contributions}

YL designed the study and performed the experiments, acquired and analyzed the data, prepared the manuscript, and read and approved the final version of the manuscript.

\section{Ethics approval and consent to participate}

The study was approved by the Ethics Committee of The First People's Hospital of Fuyang District (Hangzhou, China).
Signed informed consents were obtained from the patients and/or guardians.

\section{Patients consent for publication}

Not applicable.

\section{Competing interests}

The author declares that he has no competing interests.

\section{References}

1. Molinos M, Almeida CR, Caldeira J, Cunha C, Gonçalves RM and Barbosa MA: Inflammation in intervertebral disc degeneration and regeneration. J R Soc Interface 12: 20141191, 2015.

2. Hanaei S, Abdollahzade S, Khoshnevisan A, Kepler CK and Rezaei N: Genetic aspects of intervertebral disc degeneration. Rev Neurosci 26: 581-606, 2015.

3. Chen J, Xuan J, Gu YT, Shi KS, Xie JJ, Chen JX, Zheng ZM, Chen Y, Chen XB, Wu YS, et al: Celastrol reduces IL-1 $\beta$ induced matrix catabolism, oxidative stress and inflammation in human nucleus pulposus cells and attenuates rat intervertebral disc degeneration in vivo. Biomed Pharmacother 91: 208-219, 2017.

4. Yang RS, Wang YH, Ding C, Su XH and Gong XB: MiR-146 regulates the repair and regeneration of intervertebral nucleus pulposus cells via Notch1 pathway. Eur Rev Med Pharmacol Sci 23: 4591-4598, 2019.

5. Vo NV, Hartman RA, Patil PR, Risbud MV, Kletsas D, Iatridis JC, Hoyland JA, Le Maitre CL, Sowa GA and Kang JD: Molecular mechanisms of biological aging in intervertebral discs. J Orthop Res 34: 1289-1306, 2016.

6. Feng C, Yang M, Lan M, Liu C, Zhang Y, Huang B, Liu H and Zhou Y: ROS: Crucial intermediators in the pathogenesis of intervertebral disc degeneration. Oxid Med Cell Longev 2017: 5601593, 2017.

7. Ishibashi H, Tonomura H, Ikeda T, Nagae M, Sakata M, Fujiwara H, Tanida T, Mastuda K, Kawata M and Kubo T: Hepatocyte growth factor/c-met promotes proliferation, suppresses apoptosis, and improves matrix metabolism in rabbit nucleus pulposus cells in vitro. J Orthop Res 34: 709-716, 2016.

8. Srivastava RK, Li C, Ahmad A, Abrams O, Gorbatyuk MS, Harrod KS, Wek RC, Afaq F and Athar M: ATF4 regulates arsenic trioxide-mediated NADPH oxidase, ER-mitochondrial crosstalk and apoptosis. Arch Biochem Biophys 609: 39-50, 2016.

9. Luís A, Martins JD, Silva A, Ferreira I, Cruz MT and Neves BM: Oxidative stress-dependent activation of the eIF $2 \alpha-A T F 4$ unfolded protein response branch by skin sensitizer 1-fluoro-2,4-dinitrobenzene modulates dendritic-like cell maturation and inflammatory status in a biphasic manner [corrected]. Free Radic Biol Med 77: 217-229, 2014.

10. Luo J, Xia Y, Luo J, Li J, Zhang C, Zhang H, Ma T, Yang L and Kong L: GRP78 inhibition enhances ATF4-induced cell death by the deubiquitination and stabilization of CHOP in human osteosarcoma. Cancer Lett 410: 112-123, 2017.

11. Oh CH, Kim DY, Ji GY, Kim YJ, Yoon SH, Hyun D, Kim EY, Park H and Park HC: Cervical arthroplasty for moderate to severe disc degeneration: Clinical and radiological assessments after a minimum follow-up of 18 months - Pfirrmann grade and cervical arthroplasty. Yonsei Med J 55: 1072-1079, 2014.

12. Yang B and O'Connell GD: Effect of collagen fibre orientation on intervertebral disc torsion mechanics. Biomech Model Mechanobiol 16: 2005-2015, 2017.

13. Jin A, Shi XC, Liu Y, Sun J and Ji H: Docosahexaenoic acid induces PPAR $\gamma$-dependent preadipocytes apoptosis in grass carp Ctenopharyngodon idella. Gen Comp Endocrinol 266: 211-219, 2018.

14. Ma Y, Zhu B, Yong L, Song C, Liu X, Yu H, Wang P, Liu Z and Liu X: Regulation of intrinsic and extrinsic apoptotic pathways in osteosarcoma cells following oleandrin treatment. Int $\mathbf{J}$ Mol Sci 23, 1950, 2016.

15. Cai X, Liu Y, Hu Y, Liu X, Jiang H, Yang S, Shao Z, Xia Y and Xiong L: ROS-mediated lysosomal membrane permeabilization is involved in bupivacaine-induced death of rabbit intervertebral disc cells. Redox Biol 18: 65-76, 2018. 
16. Tschoeke SK, Hellmuth M, Hostmann A, Robinson Y, Ertel W, Oberholzer A and Heyde CE: Apoptosis of human intervertebral discs after trauma compares to degenerated discs involving both receptor-mediated and mitochondrial-dependent pathways. J Orthop Res 26: 999-1006, 2008.

17. Ha KY, Koh IJ, Kirpalani PA, Kim YY, Cho YK, Khang GS and Han CW: The expression of hypoxia inducible factor-1alpha and apoptosis in herniated discs. Spine 31: 1309-1313, 2006.

18. Zhao CQ, Jiang LS and Dai LY: Programmed cell death in intervertebral disc degeneration. Apoptosis 11: 2079-2088, 2006.

19. Wang Z, Wang D, Li Y and Zhang X: Protective effects of verapamil against $\mathrm{H}_{2} \mathrm{O}_{2}$-induced apoptosis in human lens epithelial cells. Biomol Ther (Seoul) 22: 553-557, 2014

20. Xian M, Cao H, Cao J, Shao X, Zhu D, Zhang N, Huang P, Li W, Yang B, Ying M, et al: Bortezomib sensitizes human osteosarcoma cells to adriamycin-induced apoptosis through ROS-dependent activation of p-eIF2 $\alpha / \mathrm{ATF} 4 / \mathrm{CHOP}$ axis. Int $\mathrm{J}$ Cancer 141: 1029-1041, 2017.

21. Zong Y, Feng S, Cheng J, Yu C and Lu G: Upregulated ATF4 Expression increases cell sensitivity to apoptosis in response to radiation. Cell Physiol Biochem 41: 784-794, 2017.

22. Ameri $\mathrm{K}$ and Harris AL: Activating transcription factor 4 . Int $\mathrm{J}$ Biochem Cell Biol 40: 14-21, 2008.

23. Averous J, Bruhat A, Jousse C, Carraro V, Thiel G and Fafournoux P: Induction of CHOP expression by amino acid limitation requires both ATF4 expression and ATF2 phosphorylation. J Biol Chem 279: 5288-5297, 2004.

24. Sok J, Wang XZ, Batchvarova N, Kuroda M, Harding H and Ron D: CHOP-Dependent stress-inducible expression of a novel form of carbonic anhydrase VI. Mol Cell Biol 19: 495-504, 1999

25. Fu HY, Okada K, Liao Y, Tsukamoto O, Isomura T, Asai M, Sawada T, Okuda K, Asano Y, Sanada S, et al: Ablation of C/EBP homologous protein attenuates endoplasmic reticulum-mediated apoptosis and cardiac dysfunction induced by pressure overload. Circulation 122: 361-369, 2010.
26. Yang Y, Liu L, Naik I, Braunstein Z, Zhong J and Ren B: Transcription factor C/EBP homologous protein in health and diseases. Front Immunol 8: 1612, 2017.

27. Li P, Zhou L, Zhao T, Liu X, Zhang P, Liu Y, Zheng X and Li Q: Caspase-9: Structure, mechanisms and clinical application. Oncotarget 8: 23996-24008, 2017.

28. Park GB, Choi Y, Kim YS, Lee HK, Kim D and Hur DY: ROS and ERK1/2-mediated caspase-9 activation increases XAF1 expression in dexamethasone-induced apoptosis of EBV-transformed B cells. Int J Oncol 43: 29-38, 2013.

29. Chen Y, Sun P, Bai W and Gao A: MiR-133a regarded as a potential biomarker for benzene toxicity through targeting Caspase-9 to inhibit apoptosis induced by benzene metabolite (1,4-Benzoquinone). Sci Total Environ 571: 883-891, 2016.

30. Tamaki H, Harashima N, Hiraki M, Arichi N, Nishimura N, Shiina H, Naora K and Harada M: Bcl-2 family inhibition sensitizes human prostate cancer cells to docetaxel and promotes unexpected apoptosis under caspase-9 inhibition. Oncotarget 5: 11399-11412, 2014.

31. Roberts JL, Booth L, Conley A, Cruickshanks N, Malkin M, Kukreja RC, Grant S, Poklepovic A and Dent P: PDE5 inhibitors enhance the lethality of standard of care chemotherapy in pediatric CNS tumor cells. Cancer Biol Ther 15: 758-767, 2014.

(i) $(9)$ This work is licensed under a Creative Commons Attribution-NonCommercial-NoDerivatives 4.0 International (CC BY-NC-ND 4.0) License. 